\title{
Can Modal Skepticism Defeat Humean Skepticism?*
}

\author{
Peter Hawke
}

October 28, 2016

\begin{abstract}
My topic is moderate modal skepticism in the spirit of Peter van Inwagen. Here understood, this is a conservative version of modal empiricism that insists that reasonable belief in possibility claims is sourced in a posteriori techniques that severely limit the extent to which agents can reasonably believe "exotic" possibility claims. I offer a novel consideration in support of this brand of skepticism: that modal skepticism grounds an attractive (and novel) reply to Humean skepticism. Thus, I propose that modal skepticism be accepted on the basis of its theoretical utility as a tool for dissolving philosophical paradox.
\end{abstract}

Keywords: modal skepticism, modal epistemology, modal empiricism, problem of induction.

\footnotetext{
${ }^{*}$ Working draft. Please do not cite without permission. Comments welcome! Final version to appear in Modal Epistemology After Rationalism, Synthese Library Series, R. W. Fischer and F. Leon eds., Dordrecht: Springer.
} 


\section{Introduction}

In this essay, I extol the virtues of a conservative stance regarding reasonable belief in possibility claims (not least those arrived at via a priori means, such as unfettered conceivability techniques) 墙 My primary aim is to offer a novel argument for moderate modal skepticism in the spirit of Peter van Inwagen [22], in particular in the guise of what I call the safe explanation theory of modal epistemology, a form of modal empiricism [17] $]^{3}$

The gist of my argument is this: modal skepticism provides us with simple and natural tools for disarming less benign forms of skepticism. While I believe there are general morals in the neighborhood, I presently focus on an important case study: the safe explanation theory as an antidote to Humean skepticism. I present and defend from objection a reply to Humean skepticism that has not, to my knowledge, received much attention. Stated loosely: in order for (C) one to be in a position to reasonably believe that all $X$ 's are $F$ 's, it essentially suffices that (i) one is reasonable to believe that it is possible for an $X$ to be an $F$ and (ii) one is not reasonable to believe that it is possible for an $X$ to not be an $F$. Further, (i) and (ii) hold true for an agent with (only) the sort of empirical evidence that intuitively licenses an induction by simple enumeration.

The plausibility of this reply to Humean skepticism is assured by moderate modal skepticism in conjunction with a plausible additional principle governing the interaction between possibility and reasonable belief. Properly framed, (i) and (ii) are a consequence of (a modest version of) the

\footnotetext{
${ }^{1}$ Hume and especially Descartes are often pointed to as philosophers that embrace unconstrained conceivability techniques as a guide to possibility. Recent sophisticated advocates of conceivability techniques - such as Yablo [31], Chalmers [4] and, on one reading, Bealer [2] - take great care to separate out different senses of "conceivable" and fix upon more and less promising candidates. I will relate our own discussion to some of these distinctions, often in footnotes.

${ }^{2}$ Many thanks to the editors for their constructive remarks, particularly regarding the overall focus of the present paper. Thanks to Wesley Holliday and Krista Lawlor for reading a draft of this paper, and offering useful comments. Thanks to Robert Bassett, J.T. Chipman, Huw Duffy, Michael Fitzpatrick and Krzysztof Mierzewski for their observations in response to an early version of these ideas, which led to significant improvements.

${ }^{3}$ For more on van Inwagen's epistemology of modality, including a critical discussion, see the entry in the present volume by Felipe Leon. For a critical discussion of the modal skepticism of van Inwagen [22] and Hawke [17] in tandem, see Hartl [16].
} 
safe explanation theory, and that (i) and (ii) have (C) as a consequence follows from acceptance of the principle that what one is rational to believe depends only on propositions that one is rational to consider possible (a type of relevant alternatives condition on rational belief, as explained in sect. 15. Or so I will argue.

Thus, the safe explanation theory finds significant support on grounds of theoretical utility: we can trade off a (philosophically orthodox, but ill-advised) commitment to rational access to a vast store of exotic possibility claims, in exchange for a sensible picture of inductive belief. The burden falls on the modal liberal to explain why this seemingly advantageous trade-off is unsatisfactory.

I elsewhere [17] develop the safe explanation theory as an elaboration of ideas proposed by ground-breaking fore-runners [31] [22]. In this essay, I revisit some considerations that lend modal skepticism plausibility (sects. 9 11), and flesh out certain aspects of my prior work. For I aim to recommend modal skepticism on the basis that it grounds an attractive reply to Humean skepticism, but without at least some independent support for modal skepticism, an appeal to it may appear ad hoc.

Further, pitting modal skepticism against Humean skepticism affords an opportunity to discuss one version of an important objection to the safe explanation theory: that it is too conservative. I aim to rebut this version of the objection.

For now, I turn to more expansive introductory remarks.

\section{Skepticism, benign and otherwise}

A radical modal skeptic denies that we reasonably believe any non-trivial modal claims i.e. any necessity or mere possibility claims. Accordingly, I neither know that the furniture in my lounge could be rearranged nor that an object could be both transparent and composed of iron. Alternatively, a moderate modal skeptic is one who holds that we while we have a great deal of basic, ordinary modal knowledge, our ability to establish more exotic possibility (or necessity) claims is 
importantly limited. Accordingly, I may well know that the furniture in my lounge could be rearranged, yet it is hubris (given my mundane, non-specialized evidence) to claim it possible that an object be both transparent and composed of iron. As van Inwagen puts it:

I have called this position "modal skepticism". This name was perhaps ill-chosen, since, as I have said, I think that we do know a lot of modal propositions, and in these post-Cartesian days, "skeptic" suggests someone who contends that we know nothing or almost nothing. It should be remembered, however, that there has been another sort of skeptic: someone who contends that the world contains a great deal of institutionalized pretense to knowledge of remote matters concerning which knowledge is in fact not possible. (Montaigne was a skeptic in this sense, as were, perhaps, Sextus and Cicero.) It is in this sense of the word that I am a modal "skeptic". [22, pg.69]

I too use 'modal skepticism' as shorthand for 'moderate modal skepticism'.

That modal skepticism can combat more worrying brands of skepticism should seem natural. Many classic arguments with skeptical conclusions depend heavily on an exotic possibility claim. Consider the following truncated arguments (phrased here in an especially virulent form concerning reasonable belief).

1. That it is a live possibility on my evidence that I am a massively deceived handless brainin-a-vat supports the conclusion that I am not reasonable to believe that I have hands (the argument for Cartesian skepticism).

2. That it is a live possibility on my evidence that the world could have blinked into existence two seconds ago denies me reasonable belief of the past (the argument for Russellian skepticism).

3. That it is a live possibility on my evidence that any past regularity in nature - discerned by 
observation - could suddenly cease denies me reasonable expectation of the continuation of such a regularity into the future (the argument for Humean skepticism).

A typical strategy for undermining these arguments is to accept the possibility claim embedded in the leading premise, but challenge liveness: appearances to the contrary, our evidence rules out such possibilities. The modal skeptic, on the other hand, is apt to diagnose the seeming force of these arguments as, on reflection, emanating from an unreasonable commitment to the possibility claim itself. According to her, we have an unfortunate penchant (in the philosophy classroom) to take seriously scenarios we have no epistemic right to believe are genuine possibilities.

Since Humean skepticism is our focus. ${ }^{4}$ I next (sect. 3) set the parameters for our discussion of this topic. Then (sect. 4) I more carefully state the reply to Humean skepticism that occupies our interest, and draw out some immediate objections. The bulk of the rest of the essay (sects. 5, 12 , is devoted to (hopefully instructive) replies to these objections. As a byproduct of this discussion, we compare the merits of two different formalizations (sect. 6 and 13) of our central argument. Along the way, we will find opportunity (sects. 10 and 11 to more carefully formulate and defend modal empiricism and modal skepticism, and comment on interesting connected issues. I end with a train of briefer comments: first (sect. 14), on the prospects for the modal skeptic to deal with Goodman's grue paradox (the prospects are good, but the issues delicate); and, second (sect. [15), I note the plausibility of a relevant alternatives theory of reasonable belief and its neat fit with both modal skepticism and our reply to Humean skepticism.

\section{Humean skepticism}

I now clarify Humean skepticism, only hinting at a larger issue: the problem of induction. A brief summary of the vast literature on these topics is hopeless, so my remarks are necessarily selective and sketchy. For further illumination, one may consult surveys by Norton [25] and Vickers [30].

\footnotetext{
${ }^{4}$ See Kung [21] for an argument against Cartesian skepticism from the perspective of modal empiricism.
} 
A Humean skeptic, for our purposes, denies the following:

Universal Inference: In the absence of other relevant evidence, an agent is entitled to believe that every $X$ is an $F$ on the basis of a (sufficiently large, seemingly unbiased) sample in which every $X$ is an $F$.

(I parenthesize "sufficiently large, seemingly unbiased" since it is a feature of my defense of Universal Inference that it holds even with this - typical - qualification dropped. According to this defense, Universal Inference is not grounded on probabilistic mechanisms for which a notion of ‘random selection' plays a crucial role.)

Our running (toy) example is the somewhat staid case of green emeralds. A Humean skeptic is one who denies that the following is a conclusive reason to believe that all emeralds are green: one has only ever observed green emeralds, and this is the sum total of one's relevant evidence.

I understand our Humean skeptic as similarly denying the truth of:

Singular Predictive Inference: In the absence of other relevant evidence, an agent is entitled to believe that the next $X$ they will observe will be an $F$ on the basis of a (sufficiently large, seemingly unbiased) sample in which every $X$ is an $F$.

It harmlessly simplifies our discussion to assume that universal inference and singular predictive inference are equivalent. I jointly refer to these modes of inference as enumerative induction. Our focus is therefore narrower than the broad notion of "induction" that covers any method of inference from the observed to the unobserved. We are not even immediately concerned with statistical induction, a natural generalization of enumerative induction: roughly, that an observed frequency $\sigma$ of $X$ 's that are $F$ 's (in a representative sample) entitles an agent to believe that the frequency of $F$ 's among the total population of $X$ 's is essentially $\sigma$.

Much ink has been spilt trying to put enumerative induction on firm ground. I assume for this essay that this problem has neither been satisfactorily solved nor dissolved, despite a long history of 
ingenious and theoretically rich attempts. At least, room remains for a novel and natural approach to the problem. For skeptical readers, I address this assumption more carefully in an appendix.

\section{An argument against Humean skepticism}

I claim that, assuming modal skepticism, the below argument is sound and so essentially grounds enumerative induction. For simplicity, assume in what follows that green and yellow are mutually exclusive and exhaustive properties. Let $S$ designate a logically astute rational agent that has exactly the following empirical evidence concerning the color of emeralds: $S$ has observed a notable number of emeralds, and each such emerald was green.

P1. $S$ is reasonable to believe that green emeralds are possible.

P2. $S$ is not reasonable to believe that yellow emeralds are possible.

P3. What an agent reasonably believes (given her empirical evidence) is a function of only those propositions that she reasonably considers to be possible.

C. Therefore: $S$ is both reasonable to believe that the next emerald she encounters will be green and that every emerald is green.

$\mathbf{P 3}$ is intended to express a necessary principle. P1 and $\mathbf{P 2}$ express contingencies.

This argument inspires certain immediate objections.

Objection to P2: Surely $S$ has reason to believe that yellow emeralds are "possible" in a sense that is relevant to the evaluation of her doxastic entitlements. After all, a scenario in which yellow emeralds occur seems compatible with $S$ 's limited empirical evidence (in this sense, yellow emeralds are an "epistemic possibility" for $S$ ). And it is presumably easy enough for $S$ to imagine or conceive of a situation in which she comes across a yellow emerald (so yellow emeralds are also a "conceptual possibility" for $S$ - an "epistemic possibility" in a second sense). 
Objection to P3: First, this premise is vague. Second, it might seem that there is no way to clarify it without either (i) rendering the argument invalid or (ii) rendering the premise false.

For instance, read "it is possible that $P$ " as expressing epistemic possibility, in the sense that $P$ is consistent with the agent's empirical evidence. On this reading, $\mathbf{P 3}$ seems a truism. However, the argument is not sound: as mentioned above, $\mathbf{P 2}$ seems obviously false.

Alternatively, give "it is possible that" an alethic reading. Then one might elaborate $\mathbf{P 3}$ as: if it is reasonable to believe that not-P is impossible, then it is reasonable to believe $\mathrm{P}$. This principle is uncontroversial. However, again the argument is rendered invalid: P2 does not express (nor, we can agree, does $S$ 's evidence support) reasonable belief in the impossibility of yellow emeralds.

Or one might elaborate $\mathbf{P 3}$ as: if there is no reason to believe that not-P is possible, then it is reasonable to believe not-P is impossible. But this is a version of the principle: if I have no reason to believe P, then not-P. Hence our argument reduces to an egregious argument from ignorance. That this version of $\mathbf{P 3}$ licenses fallacious reasoning may be seen with an example: consider Goldbach's conjecture. I am neither reasonable to believe that this claim is possible (for all I reasonably believe, its negation is necessarily true), nor am I reasonable to believe that its negation is possible (for all I reasonably believe, the conjecture is necessarily true). Thus $\mathbf{P 3}$, on the current reading, licenses the absurd conclusion that both Goldbach's conjecture and its negation are impossible.

\section{Real possibility}

I will reply to these objections in reverse order. But first I must clarify what I mean by "it is possible that $P$ " in the argument above.

"It is possible that $P$ " can be paraphrased as: "there exists a way things could be according to which $P$ is the case". Following David Lewis [23, pg.84], we note that acceptance of this paraphrase commits us ordinary speakers to the existence of ways things could be, some counterfactual. Like Lewis, I embrace this existential commitment, so accept realism in a broad sense. I also accept, 
like Lewis, that a way things could be $w$ is total (a "possible world") in the following sense: for any well-defined language $\mathscr{L}$, every sentence in $\mathscr{L}$ can be assigned a truth value relative to w. However, I take the standard line and resist Lewis' extravagant ontology of concrete worlds. Otherwise, I remain neutral as to what a possible world actually is.

It is also widely accepted that modal talk is shifty [19]: the modal expressions "must", "can", "might", "could" and "possibly" are apparently context-sensitive. The standard explanation: context supplies a salient set of possible worlds with respect to which such expressions are evaluated (to relate this to Lewis' above paraphrase, one might propose that context restricts the domain over which "there exists" quantifies). When "on the highway, one can only drive under 65 miles per hour" is uttered, context determines whether this sentence is evaluated relative to practical, physical, legal or moral possibility. We have linguistic resources for flagging the restricted class of possible worlds at issue: for instance, we speak of "physical possibility", "biological possibility" and "practical possibility".

I use "possibility simpliciter" to refer to the broadest category of possibility: $P$ is possible simpliciter just in case $P$ holds at some possible world in the total class of possible worlds. In the context of philosophical discussion, it is natural to take possibility simpliciter as the default reading of "it is possible that". I follow suit. Where emphasis seems necessary, I use real possibility or genuine possibility 5 Note that if $P$ is not a "real possibility" as I am using this phrase, it is no possibility at all. I avoid the technical term "metaphysical possibility", it having received a confusing variety of readings in the literature ${ }^{6}$

\footnotetext{
5 cf. Sturgeon [29, sect. 3].

${ }^{6}$ It is not uncommon for authors to equate "metaphysical possibility" with "logical possibility" or "conceptual possibility". As we discuss momentarily, I believe it is a dubious claim that mere "logical possibility" (i.e. mere logical consistency) guarantees that a set of sentences represent a possibility, and likewise for "conceptual possibility". I do agree that every real possibility can be associated with a logically consistent and conceptually coherent description.
} 


\section{Objection to P3: Reply}

The moral to glean from the objection to $\mathbf{P 3}$ (in sect. 4) is that interpretation of this premise requires care, if it is to express a plausible validity. To sharpen the discussion, I present a preliminary formalization of the argument (we will later - sect. 13- find cause to consider a second formalization of the argument. The general lessons of this section are transferable to this second formalization). Let $B \varphi$ stand for "it is reasonable for $S$ to believe that $\varphi$ ". Let $\nabla \varphi$ stand for "it is (really) possible that $\varphi$ ". Let $g$ stand for "the next emerald $S$ observes will be green".

P1. $B(\diamond g)$.

P2. $\neg B(\diamond \neg g)$

P3. $B(\diamond \varphi) \wedge \neg B(\diamond \neg \varphi) \rightarrow B \varphi$, where $\varphi$ can be replaced with any proposition letter.

C. $B g$

In the current section, I refer to the above formal expressions as "formalized P1", "formalized P2", and so on.

Formalized $\mathbf{P 3}$ represents, I believe, a plausible principle: if $P$ is the only alternative whose real possibility I am willing to commit to, then I had better believe that P. We may contrast it with more problematic principles:

1. $B(\neg \diamond \neg \varphi) \rightarrow B \varphi$

2. $\neg B(\diamond \neg \varphi) \rightarrow B(\neg \diamond \neg \varphi)$.

3. $\neg B(\diamond \neg \varphi) \rightarrow B \varphi$

1 says: "if $S$ is rational to believe that the falsity of $\varphi$ is impossible, then it follows that $S$ is rational to believe $\varphi$ ”. As noted: a plausible validity, but if we replace formalized $\mathbf{P 3}$ with this 
principle, we do not have a valid argument. There is no natural fix: replacing formalized $\mathbf{P 2}$ with $B \neg \diamond \neg g$ results in a false premise.

2 says: "if $S$ is not rational to believe that $\varphi$ is possible, then it follows that $S$ is rational to believe that $\varphi$ is impossible". As noted, 2 is a form of argument from ignorance (it is of the form $\neg B \varphi \rightarrow B(\neg \varphi))$, so we reject it.

3 says: "if $S$ is not rational to believe that $\varphi$ is possible, then it follows that $B$ is rational to reject $\varphi$ ". But 3 is not a plausible validity. 3 entails that it is inconsistent that $\neg B \diamond \neg \varphi \wedge \neg B \diamond \varphi$ (or equivalently $\neg B \diamond \neg \varphi \wedge \neg B \diamond \neg \neg \varphi)$. But this just expresses the harmless state of being undecided about a possibility (consider again $\varphi=$ Goldbach's conjecture).

If we replace formalized $\mathbf{P 3}$ with 2 or 3 in our argument against Humean skepticism, we maintain validity (though not soundness) of the argument. The relationship between formalized P3 and our alternative principles, I claim, is this: 1, 2, 3 and formalized $\mathbf{P 3}$ are all logically non-equivalent. 2 is strictly stronger than 3 , which is strictly stronger than formalized P3. That is: 2 entails 3 which entails formalized P3, but the reverse entailments do not hold. It is therefore easy to confuse an informal statement of formalized $\mathbf{P 3}$ with these other principles, by taking it as an endorsement of a stronger version of formalized $\mathbf{P 3}$ than is necessary or desirable.

Though formalized $\mathbf{P} 3$ is not obviously invalid, a worry lingers: formalized $\mathbf{P} 3$ entails that $B(\varphi)$ must hold if agent $S$ both (merely) reasonably believes that $\varphi$ is possible and rationally suspends judgment concerning $\diamond \neg \varphi$. But is it plausible that belief in $\varphi$ reasonable given only (i) at best faint direct support for $\varphi$ (namely, support for its mere possibility) and (ii) a mere lack of support for $\neg \varphi$ ? In response, I suggest that (i) and (ii) provide a misleading description of $S$ 's epistemic situation. A more fitting description, in my view: of $\varphi$ and $\neg \varphi$, only the former has the status of a live hypothesis for $S$ (using a sensible reading of " $\varphi$ is a live hypothesis for $S$ " as " $S$ rationally believes that $\varphi$ is possible"). Further, I see no way to substantiate the worry in question with a compelling theoretical proposal. For consider the proposal that formalized $\mathbf{P 3}$ is invalid precisely because it is 
a necessary condition for $B \varphi$ that $B(\neg \diamond \neg \varphi)$ must hold. But this condition is implausibly strong: I rationally believe that there is coffee in the mug before me despite not being rational to believe in the impossibility of having prepared another beverage. Consider a second proposal: that it is necessary for $B \varphi$ that the agent have conclusive reasons to reject every alternative proposition to $\varphi$ (and merely being in a position to rationally suspend judgment regarding $\diamond \neg \varphi$ does not amount to having such reasons). But this proposal is also implausibly strong. For consider: I rationally believe that there is coffee in the mug before me. An alternative proposition is that my coffee was replaced with another tan liquid (when I turned my head) by fiendish aliens using a teleportation device. This scenario is easily imagined (in a rudimentary way), and seems consistent with my basic empirical evidence. But, intuitively, consideration of such a "far-fetched" alternative does not undermine the rationality of my belief.

The immediate worries concerning (formalized) P3 are (hopefully) defused. But can a positive case be offered for its truth, especially one that is persuasive to a modal skeptic? We return to this question in sect. 15, after investigating the rationale for modal skepticism in a little more detail.

\section{7 “Possibilities" that are not possibilities}

Before replying to the objection to $\mathbf{P 2}$, I highlight another important preliminary point.

Let $M$ be some method (or class of methods) for establishing necessity claims. It is natural to speak of " $M$ necessities": those necessities that can be determined by method $M$. For instance, we may speak of logical, mathematical and conceptual necessities.

Now consider a sentence $\varphi$ such that $\neg \varphi$ is not an $M$ necessity. It is standard to refer to $\varphi$ as an " $M$ possibility". In this spirit, we may speak of logical, mathematical and conceptual "possibilities".

We should heed the advice of van Inwagen [22, pp.71-72] and resist the temptation to take our terminology here too seriously, without further argument. The idea of an $M$ necessity does not by 
itself motivate acceptance of a restricted class of real possibilities that correspond one-to-one to the " $M$ possibilities" (momentarily, we will discuss putative examples of a posteriori necessities. The denial of such a claim is a logical, mathematical and conceptual "possibility", but not a real possibility).

We should, in particular, resist an uncontested, wholesale treatment of "epistemic possibilities" as real possibilities. For instance, consider a notion of "epistemic possibility" of particular interest: that $\neg \varphi$ cannot be known a priori. Assuming a close connection between what can be ruled out on conceptual grounds - what is inconceivable - and what can be ruled out a priori, we may equate the set of epistemic possibilities with the set of conceptual possibilities.7 ${ }^{7}$ But since it is always a legitimate question for inquiry whether a given conceptual "possibility" is a real possibility, so too is it a legitimate question whether a given epistemic possibility is a real possibility ${ }^{8}$

\section{Objection to P2: Reply}

Recall P2: our agent $S$ is not rational to believe that yellow emeralds are possible. Recall also the objection to $\mathbf{P 2}$ in sect. 4. Briefly, the worry is that $\mathbf{P 2}$ is obviously false when read in a tempting way: as concerning epistemic possibility.

My initial reply should now be predictable: the intention is that "possible" is read in our antiskeptical argument as unqualified real possibility, as distinguished from notions of "epistemic possibility" according to which it is immediate that $\mathbf{P 2}$ is false. In particular, to agree that yellow emeralds are a conceptual possibility (consistent with our agent's empirical information) is not yet

\footnotetext{
${ }^{7}$ I will characterize "conceptual possibility" more carefully in sect. 9 allowing us to no longer assume this claim, but take it as a matter of definition.

${ }^{8}$ It is tempting, in the context of philosophical theorizing, to try to argue on substantive grounds that conceptual possibilities and real possibilities can be identified in some sense, in an effort to alleviate any mystery concerning what the latter are, and how it is possible to know anything about them. Nevertheless, the issues are delicate, and any such identification cannot proceed without careful qualification. For instance, a two-dimensionalist (in roughly the spirit of Chalmers [4]) might hold that ideal primary conceivability can be identified with primary possibility. In a similar vein, one might claim that a conceptual possibility given in purely qualitative terms can always be identified with a metaphysical possibility.
} 
to agree that they are a real possibility. 9

However, an important residual worry remains. Some might allege that we can locate a substantial rational connection (perhaps a fallible one) between the epistemic possibility of a yellow emerald and the real possibility of a yellow emerald, reflected in the ordinary practice of determining possibility on the basis of imagined scenarios.

From here out, we read " $\varphi$ is an epistemic possibility" as " $\neg \varphi$ cannot be known a priori", and assume the legitimate equation of this set of epistemic possibilities with the set of conceptual possibilities (we say that $\varphi$ is an epistemic possibility for agent $S$ just in case $\varphi$ is a conceptual possibility that is consistent with the agent's empirical information) ${ }^{10}$ Now, the residual worry seems rooted in support for the following:

Conceivability-Possibility Principle: That $\varphi$ is conceivable (i.e. an epistemic possibility) is conclusive reason to believe that $\varphi$ is a real possibility

However, a modal empiricist should reject this principle, unless seriously qualified. In fact, there are good reasons to be a modal skeptic, and a modal skeptic should happily accept P2. Or so I argue over the next few sections 9 - 12.

\section{The epistemology of modality}

I now sketch a general, idealized picture as to what it is to inquire into the truth of a modal claim, "possibly, $\varphi$ ” or "necessarily, $\varphi$ ”.

Fix an interpreted language $\mathscr{L}$. If it does not seem fanciful, it is a helpful heuristic to think of $\mathscr{L}$ as an ideal language corresponding to the language of thought of a conceptually ideal agent

\footnotetext{
${ }^{9}$ Indeed, this claim is plausibly a paradigm instance of the breakdown between conceivability and possibility, since it is plausible that "all emeralds are (naturally) green" is an a posteriori necessity (or, at least, this becomes clear when we properly unpack the import of inductive reasoning).

${ }^{10}$ Our sense of the term "conceivable" may thus be taken, by a two-dimensionalist, to match that of "primarily, negatively conceivable" in [4]. At this point, I deliberately leave our usage ambiguous between "ideal conceivability" and "prima facie conceivability".
} 
(one that cognizes using the full range of concepts). We assume that our language has basic logical resources: connectives, quantifiers and so on. We also assume that it contains a roster of names and natural kind terms (“George W. Bush”, "water”), with the same referents as ordinary English.

For convenience, we do not include modal operators in our language. As explained momentarily, we will talk about necessity and possibility as applying to (sets of) sentences in our language (as opposed to, say, propositions). Hence, we treat "necessarily" and "possibly" as meta-linguistic operations e.g. "possibly, $\varphi$ " is to be read as the (meta-linguistic) claim that the sentence $\varphi$ is either true or could have been true 11

Call a set of sentences from $\mathscr{L}$ a story (in $\mathscr{L}$ ). We say that a story $\mathbf{s}$ is total just in case for every sentence $\varphi$ in $\mathscr{L}$, either $\varphi$ or $\neg \varphi$ is in $\mathbf{s}$ (note a total story may contain both).

We say that a story corresponds to a way things could be $w$ just in case every sentence in that story is true of $w$. In particular, corresponding to every way $w$ is at least one total story ${ }^{12}$ We say that a story $\mathbf{s}$ is necessary just in case $\mathbf{s}$ is part of every total story that corresponds to a way $w ; \mathbf{s}$ is possible just in case $\mathbf{s}$ is part of some total story that corresponds to a way $w$.

The basic goal of an inquiry into modal matters (relative to $\mathscr{L}$ ) is to determine which stories correspond to a way things could be, and which do not (the basic goal of inquiry simpliciter is to determine which stories correspond to actuality). In terms of an individual sentence $\varphi$, we may ask if $\varphi$ is contained in all, some or none of the total stories that correspond to a way things could be.

Various familiar methods determine necessity, providing tools for categorizing stories as impossible (i.e. as not corresponding to any way things could be). Observing orthodoxy, we include:

1. Logical methods: a story that cannot be embedded in a logically consistent total story is impossible.

\footnotetext{
${ }^{11}$ As the reader has noted, throughout this essay I make use of a formal language to state formalizations of important principles and arguments. Since I freely incorporate $\diamond \varphi$ expressions in such statements, this formal language is not to be confused with the language $\mathscr{L}$ described above.

${ }^{12} \mathrm{We}$ assume here that sentences can be evaluated for truth relative to possible worlds i.e. ways things could be. Note that there is a natural sense in which a sentence $\varphi$ can be said to be "true" relative to a story $\mathbf{s}$ : namely, $\varphi \in \mathbf{s}$.
} 
2. Mathematical methods: any story that contradicts a mathematical fact is impossible.

3. General conceivability methods: any story that cannot be embedded in a conceptually coherent total story is impossible.

A story $\mathbf{s}$ is "conceptually coherent" just in case a perfectly logically astute, cognitively unbounded and conceptually expert agent cannot rule out a priori that $\mathbf{s}$ corresponds to a possibility (a "conceptual expert" has access to the full repertoire of concepts). Since I understand "a priori" to itself be an ideal notion, this amounts to: it cannot be ruled out a priori that $\mathbf{s}$ corresponds to a possibility. Hence, the terms "conceptually coherent", "conceptual possibility" and "epistemic possibility" are used interchangeably in this essay (the first being the least misleading regarding modal import).

To determine that a sentence $\varphi$ is possible seemingly requires techniques beyond those listed above. In general, we require methods for identifying a particular story $\mathbf{s}$ such that (i) $\varphi$ is part of that story, or at least entailed by it and (ii) it is sufficiently likely that s corresponds to a way things could be 13

\section{Modal rationalism and modal empiricism}

In sum, we proceed on the assumption that determining the possibility of $\varphi$ is a matter of becoming aware of a story $\mathbf{s}$ that (i) corresponds to a way things could be and (ii) that verifies $\varphi$, where story $\mathbf{s}$ verifies $P$ just in case it is a deductive consequence of $\mathbf{s}$. We say that a rational agent $S$ weakly conceives of $\varphi$ exactly when $S$ becomes aware of a story that verifies $\varphi$. We say that a rational

\footnotetext{
${ }^{13}$ Of course, if every true necessity claim were established by a cognitively ideal agent, and that agent has a principled way of establishing that this is so (i.e. that her methods for determining necessity are exhaustive) then that agent is in a position to deduce every true possibility claim. In this case, what needs to be added to the agent's repertoire, beyond techniques for determining necessity, is a means for determining what counts as an exhaustive set of methods for determining necessity. That the method of constructing a particular story that verifies $\varphi$ is a more promising candidate (for our primary method for establishing possibility claims) rests, I think, on two observations: (i) this last method seems to better reflect our actual practice of appealing to imagined scenarios to justify possibility claims, and (ii) it is decidedly non-obvious how one might establish that a set of necessity-determining techniques is exhaustive.
} 
agent $S$ strongly conceives of $\varphi$ exactly when $S$ becomes aware of a story that (i) verifies $\varphi$ and (ii) can be embedded inside a total story that is conceptually coherent.

For our purposes, a (strict) modal rationalist is one who accepts both of the following (respectively, a metaphysical principle and an epistemic principle).

Ideal Conceivability-Possibility Principle: If rational agent $S$ strongly conceives of $\varphi$ then it follows that $\varphi$ is possible ${ }^{14}$

Practical Conceivability-Possibility Principle: If rational agent $S$ weakly conceives of $\varphi$ in such a manner that no incoherence is detectable by $S$, then $S$ is reasonable to believe that $\varphi$ is possible.

A modal empiricist, as we understand that position here, rejects both of these principles.

What I have in mind by "being unable to detect incoherence" is this: the union of that story with every necessity that the agent knows of seems, to the agent, to be a logically consistent story.

The practical C-P principle qua methodological touchstone (as opposed to the ideal C-P principle) reflects an admission that we are cognitively limited beings, with no hope of checking once and for all that a story can be extended into a conceptually coherent total story. The practical C-P principle is an instance of what I'll call a coherence detection principle. Such a principle is "negative": it claims that having no reason to think that $\mathbf{s}$ is incoherent is conclusive reason to accept that $\mathbf{S}$ is coherent 15

With all this mind, here are two lines of argument for rejecting (strict) modal rationalism. Note that the second targets the practical C-P principle in a manner that is independent of the truth of the ideal C-P principle (i.e. I intend this argument to have bite even if the latter is assumed).

\footnotetext{
${ }^{14}$ Notice that our particular usage in this essay of the term "conceivable" makes it plausible that the ideal C-P principle captures the spirit of modal rationalism in sufficient generality. For observe that the ideal C-P principle, by our lights, is equivalent to: if $\varphi$ is necessary then it follows that $\varphi$ is knowable a priori.

${ }^{15}$ The distinction between the ideal C-P principle and practical C-P principle is closely related to the distinction between ideal and prima facie conceivability introduced by Chalmers [4].
} 
1. Existence of a posteriori necessities: a necessary sentence $\varphi$ whose modal status can only be established a posteriori is a counter-example to the ideal C-P principle (for in this case $\neg \varphi$ is strongly conceivable but not possible). As is frequently discussed, Kripke [20] identifies compelling instances of this phenomenon, including identity statements involving natural kinds ("heat is the motion of molecules") and claims about the origins of objects ("George W. Bush is the biological son of Barbara Bush"). The appeal of these examples rests on acceptance of essentialist doctrines that are not beyond dispute (cf. [6]). Nevertheless, I put aside such debate in this essay. Kripke's examples strike me, and many others, as compelling.

2. Doubts about the reliability of unconstrained conceivability techniques: On the face of it, the practical C-P principle constitutes an instance of the fallacy from ignorance: that I have no reason to believe that I am not strongly conceiving of $\varphi$ is offered as a reason to conclude that I am strongly conceiving of $\varphi$. This worry would be quieted with a special rationale for taking this principle as a reliable guide to conceptual coherence. But it is hard to locate this rationale. For one thing, an ordinary individual's means for constructing nonactual stories (that is, via their imaginative abilities) is notoriously unconstrained. It is easy to construct a fictional story according to which any number of modally controversial claims hold: George W. Bush is a soviet automaton; teleportation occurs; time travel occurs; difficult mathematical problems are solved, and so forth. It would help if two facts could be established: (i) when considering a story that verifies $\varphi$, ordinary agents are practically able to fill out that story in enough detail so that all relevant facts connected to $\varphi$ are settled, for comparison with the agent's store of known necessity claims; and (ii) ordinary agents tend to have a fairly comprehensive set of known necessity claims. But both (i) and (ii) seem to be false ${ }^{16}$ The stories produced by (the imagination of) ordinary agents tend to be massively under-described relative to a total story, and the details are often difficult to fill out. And

\footnotetext{
${ }^{16}$ See [14] for a careful discussion of the implications for modal rationalism of the practical incompleteness of the stories we can construct.
} 
in general it seems no easy matter for ordinary agents to establish necessity claims. An example (following Yablo) illustrates both points [31]: it is easy to construct a story in which Goldbach's conjecture is true: imagine a team of mathematicians that prove the result and their ensuing adventures as they share the news. But it is difficult to see how to fill out every relevant detail of this story. How, for instance, are we to describe the details of the mathematician's proof? Relatedly, the store of known necessities with which to assess this story is generally meager. An ordinary agent lacks knowledge of countless mathematical necessities, not least the verdict on Goldbach's conjecture. In all, it is foolhardy to conclude from this partial story that Goldbach's conjecture is possible.

A rationalist that accepts the force of the above objections can retreat to a moderate modal rationalism by rejecting one or both of the above C-P principles. Indeed, consideration of a posteriori necessities has proven so compelling that many leading modal rationalists are properly classed as moderates [2, 4].

At the risk of simplifying, at least two (related) ideas for restricting the ideal C-P principle while maintaining a substantial role for a priori reasoning - have proven influential ${ }^{17}$ First, it is claimed that the strong conceivability of $\varphi$ entails the possibility of $\varphi$ if $\varphi$ is a purely "qualitative" claim (i.e. free of names, natural kind terms and the like) ${ }^{18}$ Second, it is proposed that, for every $\varphi$ that is necessary, if $\square \varphi$ is not knowable a priori then at least it is knowable a priori that $\square \varphi$ follows if $\varphi$ is true.

Amending the ideal C-P principle in this way is a significant retreat: "moderate modal rationalism" may just as well be called "moderate modal empiricism". Note further that embracing moderation at the level of the ideal principle does not yet resolve the concerns about the practical C-P principle. Suppose we accept that conceivability entails possibility at the level of "purely qual-

\footnotetext{
${ }^{17}$ See [29] for a careful and comprehensive evolution of positions one might take on in response to Kripkean considerations.

${ }^{18}$ Compare this to Bealer's notion of semantic stability [2] pg. 72] and, in the terminology of Chalmers [4], a sentence whose primary intension coincides with its secondary intension.
} 
itative" descriptions. This does nothing to allay the worry that ordinary imaginings (often utilized to construct stories that feature names, natural kinds, mathematical claims and so on) are unconstrained in their content, severely limited in their scope and regulated by few known necessities.

Indeed, I suggest that one who accepts the force of Kripkean considerations, but wishes to maintain a version of the practical C-P principle, faces an apparent dilemma if choosing between the practical C-P principle (as presented above) and a conservative refinement of the following form:

The Safe Explanation Principle: If rational agent $S$ is aware of story s such that $\mathbf{S}$ verifies $\varphi$ and every sentence in $\mathbf{s}$ is reasonably believed to be either (i) part of a modally safe base or (ii) a deductive consequence of that story's modally safe base, then $S$ is reasonable to believe that $\varphi$ is possible.

A modally safe base is a set of sentences such that the conjunction of those sentences expresses an uncontroversial possibility claim. ("Controversial" may be understood as relative to $\diamond \varphi$, the possibility-claim that one intends to establish.) The rationale for adopting this refined practical principle is as follows: Kripke's a posteriori necessities teach us that it is easy to (weakly) conceive of claims that are either known to be impossible or at least controversial. It is easy to construct a story s according to which water is $X Y Z$ (or George Bush's mother is Greta Garbo). Now, if the actual nature of water is known (or Bush's actual origin) then the antecedent of the original practical C-P principle is not satisfied by $\mathbf{s}$, for the union of $\mathbf{s}$ with the agent's known necessities is not consistent. But what if these facts are not known? In this case, at best, the agent knows that the claims in question are modally controversial (for, if water's composition is not known, it is at least knowable that either it is necessary that water is $X Y Z$ or it is necessary that it is not). But an admittedly controversial story that verifies $\varphi$ obviously does not support reasonable belief in $\diamond \varphi$. Thus, s cannot be taken as support for: it is possible that water is $X Y Z$. Similarly, imagining a newspaper report that the Goldbach conjecture is true does not support the possibility that the 
conjecture is true, since it is recognizably controversial whether it is possible to correctly report this claim. ${ }^{19}$ All this suggests a simple solution: reject an unconstrained practical C-P principle, and propose instead that conceivability only acts as a guide to possibility when one constructs stories from "safe" materials.

Here, however, is the dilemma 20 First horn: the truth of the original, unrefined practical C-P principle now seems particularly in doubt, for a principled reason: it entails that belief in a possibility claim is reasonable even on the basis of a story that is recognized as modally controversial. Second horn: But the safe explanation principle might seem so strict that it fails to account for mundane, indisputable possibility knowledge. I know that there is currently no coffee in my mug $(\neg \varphi)$. On what basis can I conclude $\diamond \varphi$, that there could be? It is of course easy to construct a story according to which I go to the kitchen and prepare the coffee. But is this story built from modally uncontroversial claims, relative to $\diamond \varphi$ ? If I put on hold the (in context) controversial stipulation that the mug in the story is my mug, then the story apparently no longer verifies $\varphi$ (for whatever remains could just as well concern some other mug). But upon what modally safe base can one restore the verification of $\varphi$ ? The proposal to consult one's known necessity claims seems of little help: if my mug has essential properties, then I do not know what they are 21

\section{Modal skepticism again}

A natural way to resolve the dilemma is to embrace a robust empiricism: empirical knowledge is not only required to resolve the status of a posteriori necessities, but also provides an extensive base of modally uncontroversial claims (along with any known a priori necessity claims) upon which to build stories for establishing both mundane and more unusual possibility claims. This combination of empiricism with the safe explanation principle is what I previously called the safe

\footnotetext{
${ }^{19}$ Compare Yablo's discussion of undecidable claims in [31, sect. XI].

${ }^{20}$ This dilemma is a generalization of that which I present for van Inwagen in [17].

${ }^{21}$ For further discussion of the tension between conceivability techniques and de re possibility knowledge, see [27].
} 
explanation theory of modal epistemology [17].

For this theorist, a possibility claim is (recursively) rendered modally uncontroversial just in case it is either supported by the safe explanation principle, or is directly supported on the basis of empirical investigation. Our safe explanation theorist is therefore committed to (i) the existence of empirical methods that can establish modal claims and (ii) combinatory methods that reliably allow one to combine established possibility claims into larger stories ${ }^{22}$ Here is a list of potential methods that are of this sort:

1. Actuality Principle: If it is reasonable to believe that $\varphi$, it is reasonable to believe that $\varphi$ is possible.

2. Similarity Principle: If it is reasonable to believe that $F$ is a possible property for object $o_{1}$ and $o_{2}$ appears relevantly similar to $o_{1}$, then it is reasonable to believe that $F$ is a possible property of $o_{2}{ }^{23}$

3. Abduction: If part of the best explanation for established fact $\varphi$ is that $\psi$ is possible (or necessary), then it is reasonable to believe that $\psi$ is possible (or necessary) 24

4. Property Recombination: If it is reasonable to believe that object $o$ could exist and that property $F$ could be instantiated, then it is reasonable to believe that $o$ could be $F$.

5. Strong Recombination: If it is reasonable to believe that $\varphi$ is possible and that $\psi$ is possible and there is no reason to think either is necessary, then it is reasonable to believe that $\varphi \wedge \psi$, $\varphi \wedge \neg \psi, \neg \varphi \wedge \psi$ and $\neg \varphi \wedge \neg \psi$ are all possible.

6. Modest Recombination: If it is reasonable to believe that $\varphi$ is possible, that $\psi$ is possible and that $\varphi$ and $\psi$ describe independently existing states of affairs, then it is reasonable to

\footnotetext{
${ }^{22}$ That I ignored the subtleties involved with the question of combination represents a lacuna in my presentation of the safe explanation theory in [17], one I hopefully begin to fill here.

${ }^{23} \mathrm{cf}$. Hawke [17, Sect. V] and the chapter by Sonia Roca-Royes in the present volume.

${ }^{24}$ See [3] and [10] for careful discussions of the role of abduction in modal epistemology.
} 
believe that $\varphi \wedge \psi, \varphi \wedge \neg \psi, \neg \varphi \wedge \psi$ and $\neg \varphi \wedge \neg \psi$ are all possible.

The plausibility of modest recombination depends on an account of independent existence (and how it is established). We make no commitments on this front, so leave this phrase as a suggestively named "black box" restriction. To give a sense of its potential import: one might take the independence of $\varphi$ and $\psi$ as established by the fact that it is known that each held respectively in virtue of state of affairs $s_{1}$ and $s_{2}$, and $s_{1}$ and $s_{2}$ were located in disjoint regions of space-time (cf. [24, pg. 88]) 25

Of these principles, only the actuality principle seems entirely beyond philosophical dispute.

We now have tools on the table to refine our account of modal skepticism. In particular, we can grade the severity of modal skepticism associated with a safe explanation theory according to the extensiveness of the list of empirical methods accepted by that theorist. A radical modal skeptic accepts only the actuality principle. A very modest modal skeptic accepts every principle on the above list. This last variant is too weak for van Inwagen [22]: property recombination justifies the possibility of transparent iron, exactly the kind of possibility claim that van Inwagen is suspicious of accepting too quickly.

For our part, I settle on an account of safe explanation theory that accepts the actuality principle, the similarity principle, abduction and modest recombination (of some form). I do not defend this choice beyond noting the intuitive pull of these principles. However, I reject property recombination and strong recombination. For one thing, I do not see any positive reason for accepting these principles. For another, they have apparent counter-examples. According to property recombination, it is reasonable to believe that Barbara Bush could have been the mother of Barack Obama. According to strong recombination, since my car is black-all-over and my car is not crimson-all-

\footnotetext{
${ }^{25}$ A principle of recombination has found support in the literature, notably by David Lewis [24, pp.86-92] and David Armstrong [1], though they understand the principle foremost along metaphysical lines. Of the principles of recombination I list, modest recombination is closest in statement to the principles that Lewis and Armstrong endorse, though the details of their accounts results in their versions of the principle having more elaborate consequences than I would be prepared to endorse. Lewis' inspiration is Hume's denial of necessary connections between distinct existences, while Armstrong's inspiration traces to Wittgenstein's Tractatus.
} 
over, it is possible for my car to be black-all-over and crimson-all-over.

\section{Modal skepticism supports P2}

We return to our argument against Humean skepticism, and consider a modal skeptic's assessment of formalized P2. Given that the only relevant evidence available to $S$ is an uninterrupted history of observations of green emeralds, is $S$ reasonable to fail to believe that a yellow emerald is possible? Is $S$ reasonable to fail to believe that the next emerald she observes could possibly be yellow?

In both cases, I propose the natural answer is 'yes'. Our modal skeptic accepts a possibility claim $\diamond \varphi$ just in case she can bring to mind a story that verifies $\varphi$ on a modally uncontroversial base. No such base seems available for the claim that a yellow emerald is possible, nor the claim that it is possible that the next emerald to be observed is yellow. To see this, consider the tools available to the modal skeptic. Since yellow emeralds have not been observed, the actuality principle does not secure their possibility. Since the only relevant evidence is an observation stream of green emeralds, the agent does not have available evidence of relevantly similar objects that could be yellow, so the similarity principle is not applicable. It is hard to see how positing the possibility of yellow emeralds could aid in the best explanation of the agent's observations of green emeralds, so abduction cannot be deployed. And since we have only modest recombination, the agent cannot conclude that yellow emeralds are possible simply on the basis of the existence of both emeralds and yellow objects.

\section{Does modal skepticism support P1?}

Modal skepticism is a conservative modal epistemology with certain theoretical advantages: limiting the scope of what is rightly considered possible better fits our epistemic practice.

Conservativeness can be its own source of difficulty, however. A general source for concern is 
that a modal skeptic systematically under-estimates what possibility claims are reasonably believed.

A version of this difficulty arises in our context. Recall the formalization introduced in sect. 6:

P1. $B(\diamond g)$.

P2. $\neg B(\diamond \neg g)$

P3. $B(\diamond \varphi) \wedge \neg B(\diamond \neg \varphi) \rightarrow B \varphi$, where $\varphi$ can be replaced with any proposition letter.

C. $B g$

where $g$ is the claim that the next emerald to be observed is green. Call this formalization the next emerald reading.

Does a modal skeptic have resources to endorse P1? Or are her resources too impoverished to establish this uncontroversial possibility claim?

There is room for real doubt here. On the next emerald reading, P1 is the claim that it is reasonable for the agent to believe that the next emerald she observes could be green. Suppose that it is known that this next emerald is not one of those already observed by the agent. Our agent clearly has safe empirical grounds (namely, the actuality principle) for claiming that every emerald already observed could be green. But on what empirical grounds (short of induction) can the agent project this possibility onto an unobserved emerald?

One legitimate strategy for the modal empiricist is to try establish P1 using the full repertoire of empirical methods discussed in the previous section. In particular, the modal empiricist might suggest that the possibility that the next observed emerald is green follows from abductive reasoning, or on the basis of the relevant similarity between observed emeralds and unobserved emeralds.

This strategy is worth examination, though I admit to doubts about its prospects. I am wary of hand-waving appeals to abductive reasoning. Further, the reader would be correct to note that the similarity principle is a close relative of enumerative induction. It is plausibly a weakening 
of singular predictive inference: if every observed object of a certain type $T$ has modal property $F$, then it reasonable to conclude that further object $o$ of type $T$ also has that modal property. So an appeal to the similarity principle to ground $\mathbf{P 1}$ might seem a significant retreat: we would be relying on a type of enumerative induction to ground enumerative induction in full generality.

Consider a second strategy: offer a different formalization of our argument against Humean skepticism, with a reading of $\mathbf{P 1}$ that is easier to defend. The next emerald reading involves us in premises about the next emerald to be observed, a particular emerald referred to by description. I attempt a second formalization that avoids reference to any particular emerald. Let emerald $(x)$ stand for " $x$ is an emerald" and green $(x)$ stand for " $x$ is green".

P1. $B(\diamond \exists x($ emerald $(x) \wedge$ green $(x)))$.

P2. $\neg B(\diamond \exists x($ emerald $(x) \wedge \neg$ green $(x)))$

P3. $B(\diamond \exists x(\varphi(x) \wedge \psi(x))) \wedge \neg B(\diamond \exists x(\varphi(x) \wedge \neg \psi(x))) \rightarrow B(\forall x(\varphi(x) \rightarrow \psi(x)))$ for any $\varphi(x)$ and $\psi(x)$.

C. $B(\forall x(\operatorname{emerald}(x) \rightarrow \operatorname{green}(x)))$

Call this the kinds reading. It lacks the simplicity of the next emerald reading. Nevertheless, the kinds reading better capitalizes on a leading idea behind our anti-Humean argument: that the evidence establishes the possibility of a certain kind of emerald, while failing to establish the possibility of any other kind. According to the kinds reading, P1 states that a green emerald is reasonable to consider possible; $\mathbf{P 2}$ states that a non-green emerald is not reasonable to consider possible; and $\mathbf{P 3}$ licenses an inference to the conclusion that all emeralds are green.

One downside of this formalization is that $\mathbf{P 3}$ is harder to parse and so harder to discuss and defend. I remark that similar points may be made with respect to this version of $\mathbf{P 3}$ as were made with respect to the principle $B(\diamond \varphi) \wedge \neg B(\diamond \neg \varphi) \rightarrow B \varphi$ in sect. 6. Once distinguished from superficially similar but obviously dubious principles, it has (I believe) intuitive appeal. 
The kinds reading allows the modal skeptic to defend $\mathbf{P 1}$ and $\mathbf{P 2}$ using minimal resources. The actuality principle can be used to establish P1. And there remains no obvious way to establish $\diamond \exists x($ emerald $(x) \wedge \neg$ green $(x))$ using only the actuality principle, similarity principle, abduction or modest recombination.

Settling which formalization is ultimately superior is a matter I leave for elsewhere. Our purpose here is only to note that the modal skeptic has to work to establish P1, and various avenues present themselves.

\section{The grue paradox}

No defence of enumerative induction is complete without mention of Goodman's new riddle of induction [13]. The modal skeptic has intriguing resources for tackling the grue paradox. An interesting observation: the next emerald reading of our argument against Humean skepticism provides a more elegant reply to this problem than the kinds reading (giving pause to the those convinced by the previous section that the latter is obviously superior).

Fix $t$ to be the time at which rational agent $S$ will observe her next emerald. Object $x$ is grue just in case: if the time at which $x$ is observed is less than $t$, then $x$ is green at the time of its observation; and if the time at which $x$ is observed is greater than or equal to $t$, then $x$ is blue at the time of its observation. To overcome the new riddle of induction, we require that the reasoning underpinning our argument against Humean skepticism does not allow us to conclude that the next emerald to be observed is grue, nor that every emerald is grue.

On the next emerald reading, this outcome is readily defensible. Consider again the general reasoning pattern: $B \diamond \varphi, \neg B \diamond \neg \varphi$, therefore: $B \varphi$. The soundness of this reasoning when $\varphi$ is replaced with "the next emerald to be observed is grue" requires, as per the first premise, that it is reasonable for $S$ to believe, on her evidence, that it is possible that the next emerald to be observed is grue. Let us assume, however, that it is correct by the lights of the modal skeptic that it is not 
reasonable for $S$ to believe that it is possible that the next emerald to be observed is blue. Now, by definition, the claim that the next emerald to be observed by $S$ is grue at the time of observation is equivalent to the claim that this next emerald is blue at the time of its observation (since by definition of $t$ the time of observation of this emerald is greater than or equal to $t$ ). But the modal skeptic, we suppose, is not reasonable to accept the possibility of the latter and, therefore, neither the possibility of the former. Hence, the first premise cannot be modified to the case of grue and remain true, and so the argument cannot be recreated in the case of grue.

The kinds reading, however, requires more care. Here, it needs to be established that, for the modal skeptic, the following reasoning is not sound:

$$
\begin{aligned}
& B(\diamond \exists x(\text { emerald }(x) \wedge \operatorname{grue}(x)) ; \\
& \neg B(\diamond \exists x(\text { emerald }(x) \wedge \neg \operatorname{grue}(x)) ;
\end{aligned}
$$

therefore: $B(\forall x($ emerald $(x) \rightarrow \operatorname{grue}(x)))$.

Since every observed emerald by $S$ has been grue, it is hopeless to deny the first premise. So, on the kinds reading, the modal skeptic needs to deny the second premise. This is equivalent to endorsing the following (where, for simplicity, we consider being a blue emerald and being a not-green emerald to be equivalent):

It is reasonable for $S$ to believe that: possibly, either there exists an emerald that is observed before time $t$ but is not green at the time of observation, or there exists an emerald that is observed after time $t$ and is green at the time of observation.

I believe that a plausible (if incomplete) story can be told as to why a modal skeptic should accept this. Note that the modal skeptic can accept the foregoing if she accepts the following:

It is reasonable for $S$ to believe that: possibly, there exists an emerald that is observed after time $t$ and is green at the time of observation. 
I claim that the modal skeptic can accept this on the basis of (i) $S$ 's observation of green emeralds before time $t$, (ii) time-independence of (certain) possibility claims and (iii) modest recombination. $S$ 's observations confirm the existence of a green emerald before time $t$. By the actuality principle, she reasonably believes that it is possible that a green emerald exists before $t$. If she correctly regards a possibility claim of this type as time-independent, she may reasonably accept the possibility that a green emerald exists at or after $t$. Now, suppose that it can be established that the state of affairs of observing an emerald and that of an emerald being a certain color are independently existing states of affairs. Then $S$ may conclude by modest recombination that it is possible that there exists an emerald that is observed after time $t$ and is green at the time of observation.

This response to the threat of a grue paradox involves various undefended commitments. To this extent, the next emerald reading has an immediate theoretical advantage over the kinds reading.

\section{Support for P3: a relevant alternatives theory of rational belief}

With all our cards on the table, we return to the following question: is there a positive case for $\mathbf{P 3}$ in our argument against Humean skepticism, in particular one that will appeal to a modal skeptic? We have seen a rough statement of $\mathbf{P 3}$ in sect. 4. what an agent reasonably believes (given her empirical evidence) is a function of only those propositions that she reasonably considers to be possible. We have also seen two formal principles that are inspired by this claim:

- Principle A: $B(\diamond \varphi) \wedge \neg B(\diamond \neg \varphi) \rightarrow B \varphi$

- Principle B: $B(\diamond \exists x(\varphi(x) \wedge \psi(x))) \wedge \neg B(\diamond \exists x(\varphi(x) \wedge \neg \psi(x))) \rightarrow B(\forall x(\varphi(x) \rightarrow \psi(x)))$

A robust approach would ground $\mathbf{P 3}$ in (or, perhaps, explicate it as) a plausible general principle governing rational belief and real possibility, and offer a comprehensive theoretical defense of that general principle (locating it within broader traditions in the literature). I venture some preliminary thoughts along these lines. In what follows, we identify justified belief with rational 
belief, and concentrate on issues of propositional justification, as opposed to doxastic justification. Now consider:

Possibility-based RA condition: Rational agent $S$ is reasonable to believe $\varphi$ only if $S$ has evidence that rules out every alternative $\psi$ to $\varphi$ such that it is reasonable for $S$ to believe that possibly $\psi$.

More formally:

$B \varphi$ only if $S$ has evidence that rules out every alternative $\psi$ to $\varphi$ such that $B(\diamond \psi)$.

(An alternative to $\varphi$ is a proposition $\psi$ that entails $\neg \varphi$.)

The above proposal - for a necessary condition on reasonable belief - is similar in spirit and form to a relevant alternatives condition on knowledge (cf. [7]): it amounts to the claim that only relevant alternatives to $\varphi$ need to be ruled out by the agent's evidence for that agent to be rational to believe $\varphi$, where the relevance of an alternative depends on what the agent reasonably believes to be really possible.

The possibility-based RA condition is too weak to guarantee the validity of formalized P3. Further, if the RA condition is strengthened to be both a necessary and sufficient condition on rational belief, then we have a theory of belief that is too strong, validating the following principle:

$$
\neg B(\diamond \neg \varphi) \rightarrow B \varphi
$$

We encountered and rejected this principle in sect. 6 , on the basis that it entails that $\neg B \backslash \neg \varphi \wedge$ $\neg B \diamond \varphi$ is inconsistent. I therefore suggest the following:

Possibility-based RA principle: $B \varphi$ if and only if (i) $S$ has evidence that rules out every alternative $\psi$ to $\varphi$ such that $B(\diamond \psi)$ and (ii) $S$ 's evidence supports the truth of $\diamond \varphi$. 
That is: rational belief in $\varphi$ is a matter of having ruled out the alternatives to $\varphi$ that are reasonable to consider possible, along with having positive reason to think that $\varphi$ is itself a possibility. This account is a good fit with Principle A, which amounts to: if there is conclusive reason to believe $\diamond \varphi$ and there is no relevant alternative to $\varphi$, then it is reasonable to believe $\varphi$. This account is also a good fit with Principle B. For Principle B may be understood as the claim that: if it is a reasonable to accept the possibility that there is evidence that supports that all $\phi$ 's are $\psi$ 's (where we presume that $\exists x(\varphi(x) \wedge \psi(x))$ is supporting - if not conclusive - evidence for $\forall x(\varphi(x) \rightarrow \psi(x))$, merely as a matter of the former being a highly probable observable consequence if the latter hypothesis is accepted), and there there is no relevant alternative to $\forall x(\varphi(x) \rightarrow \psi(x))$, then it follows that it is reasonable to believe that every $\phi$ is a $\psi$.

Support can be found for the possibility-based RA principle in its capacity to explain certain ordinary judgments. Example 1: consider the "coffee" example from sect. 6. though I can apparently conceive of the situation that my coffee has been replaced (via teleportation) by fiendish aliens, the compatibility of this "far-fetched" situation with my empirical information does not (intuitively) undermine the reasonableness of my belief that there is coffee in my cup. Rather, it is enough that the evidence rules out certain "realistic" alternatives: that I accidentally ordered another drink; that no-one has knocked my cup over; and so on. Example 2: I enter the room and notice a drinking glass on the table that appears to be filled with clear liquid. Intuitively, to be in a position to reasonably believe that there is water in the glass, I require empirical evidence that rules out certain "realistic" alternatives: that there is vodka in the glass, that there is nothing in the glass etc. However, I do not need to rule out "far-fetched" alternatives such as that incognito visitors from twin-earth have deposited water-indistinguishable XYZ in the glass (note that, if we are on board with Putnam [26], XYZ and twin-earth are - apparently - conceivable), even though it seems rash to commit to the impossibility of water-indistinguishable XYZ. Example 3: I am dismayed to find that the last cookie has vanished. Intuitively, in order to reasonably believe that Sam ate the cookie 
I need sufficient evidence to rule out certain "realistic" alternatives: that I ate the cookie, that a rat ate the cookie etc. I do not need evidence that rules out "far-fetched" alternatives such as that a miracle occurred and the cookie spontaneously annihilated (despite the apparent conceivability of miracles, as is typically accepted in, for instance, philosophical discussion of counterfactuals). Yet it would be epistemically immodest to claim that miracles are impossible. Example 4: I pour a glass of milk from the refrigerator. In order to reasonably believe that it is safe to drink I must rule out certain "realistic" alternatives: that the milk did not expire a week ago, that nobody laced the milk with cyanide etc. I do not need to rule out "far-fetched" alternatives such as that pure, fresh milk will act as a poison for the human body over the course of the next half-an-hour, in contrast to its typical "nourishing" qualities (compare Hume's famous observation that it is conceivable that bread need not nourish in the future, though it has done so in the past). Yet it would be epistemically immodest to claim that this scenario is impossible.

How is the data best explained? What counts as a promising explanation depends on one's theoretical commitments. If one accepts that possibility knowledge is acquired "cheaply", perhaps via crude conceivability methods, then one is presumably forced to concede that the "far-fetched" alternatives in the above examples have been established as real possibilities for the agent. Such a theorist will have to explain why these possibilities do not undermine the agent's rational belief despite (apparently) being compatible with her empirical information. (It is far from clear how such a project is best carried out.) On the other hand, suppose that one does not accept that possibility knowledge is cheaply acquired, via rudimentary conceivability techniques or otherwise. In that case, the above "far-fetched" alternatives are exotic enough to license hesitation: it hardly seems that the agent is in a position to regard them as real possibilities, unlike the mundane "realistic" alternatives.

A modal skeptic - in particular, a safe explanation theorist - is of the second variety: for her, there is a striking correlation in the above examples between, on one hand, the "realistic" alter- 
natives and the alternatives established as real possibilities and, on the other hand, between the "far-fetched" alternatives and the alternatives whose real possibility remains in question ${ }^{26}$ Thus, since the possibility-based RA principle systematizes these simple observations, it is supported for the modal skeptic on the grounds of its explanatory power ${ }^{27}$

\section{Conclusion}

We have presented an argument against Humean skepticism, proposing that the reasonableness of enumerative induction is a special case of the claim that a rational agent need only take seriously propositions that she has reason to think are really possible. This line is given substance by defending modal skepticism, and with it the claim that it is not reasonable for an agent to believe that yellow emeralds are possible given only the evidence available in the context of a reasonable enumerative induction. We found opportunity to give general characterizations of modal epistemology, modal rationalism, modal empiricism and modal skepticism. Besides sketching some initial

\footnotetext{
${ }^{26}$ Here is another, perhaps tempting, explanation of the coffee case. Though it is consistent with my empirical information that aliens have replaced my coffee, I can conclude on inductive grounds that this is not so: for it has never been my experience in the past that my coffee has mysteriously been replaced with another liquid before I have had a chance to drink it. Given the main contentions of the current paper - that simple enumeration is a special case of the kind of "possibility reasoning" I use to explain the case in the main text - it comes as no surprise that inductive reasoning comes to mind when considering the example. At any rate, the example is easily altered so as to be immune to an explanation via induction: consider instead the alternative that my coffee has been replaced by aliens (via teleportation), who will then (to avoid giving the game away) replace this second liquid with coffee before it reaches my lips. Simple enumeration cannot - in any straightforward way - rationalize the rejection of this alternative. For my empirical information did not verify that such a double-replacement did not occur during my past experiences of drinking coffee.

${ }^{27}$ Support for our argument against Humean skepticism (understood broadly) does not, in fact, require that the modal empiricist accept exactly the possibility-based RA principle. Suppose, for instance, that our empiricist accepts the need for an RA approach to rational belief, but is sufficiently impressed by the Goldman-Ginet barn cases in [12] that they decide that the most plausible RA principle is the following: $B \varphi$ if and only if (i) $S$ has evidence that rules out every alternative $\psi$ to $\varphi$ such that $S$ is reasonable to believe that $\psi$ is a nearby possibility and (ii) $S$ 's evidence supports that $\varphi$ is a nearby possibility. Now, suppose that $\mathbf{P 1}$ is true - our empiricist reasonably believes that green emeralds are really possible - and that $\mathbf{P 2}$ is true - our empiricist does not reasonably believe that yellow are emeralds are really possible. Now, note that (plausibly) the empirical support for $\mathbf{P 1}$ amounts to support for a stronger claim: that our empiricist reasonably believes that there are green emeralds. It follows, by a version of the actuality principle, that she reasonably believes that green emeralds are a nearby possibility. Further, it follows from $\mathbf{P} 2$ that she does not reasonably believe, in particular, that yellow emeralds are a nearby possibility. Hence, our empiricist's RA principle delivers the desired result: it is not reasonable for her to believe that there are yellow emeralds.
} 
considerations in favour of modal skepticism, our discussion of Humean skepticism itself provides notable support for modal skepticism, demonstrating the theoretical utility of modal skepticism as an antidote to paradox. As the last few sections make apparent, there are outstanding details that a modal skeptic must fill in for her picture to be complete (and responsive to challenges posed by the strictness of modal skepticism and the grue paradox). We have sketched possible avenues for reply to such worries, but many details remain to be explored.

\section{References}

[1] Armstrong, D., A Combinatorial Theory of Possibility, Cambridge University Press (1989)

[2] Bealer, G., "Modal Epistemology and the Rationalist Renaissance", in Conceivability and Possibility, Gendler, Hawthorne eds., Oxford University Press (2002)

[3] Biggs, S., “Abduction and Modality”, Philosophy and Phenomenological Research, Vol. LXXXIII No. 2, pp.283-326 (2011)

[4] Chalmers, D. J., “Does Conceivability Entail Possibility?”, in Conceivability and Possibility, Gendler, Hawthorne eds., Oxford University Press (2002)

[5] Chalmers, D.J.,“Epistemic Two-Dimensional Semantics”, Philosophical Studies 118.1-2, pp. 153-226 (2004)

[6] Della Rocca, M., "Essentialism versus Essentialism", in Conceivability and Possibility, Gendler, Hawthorne eds., Oxford University Press (2002)

[7] Dretske, F., “The Pragmatic Dimension of Knowledge”, Philosophical Studies 40, pp. 363$378(1981)$ 
[8] Earman, J., Bayes or Bust? A Critical Examination of Bayesian Confirmation Theory, MIT Press (1992)

[9] De FinetTi, B., "Foresight: Its Logical Laws, Its Subjective Sources", in Annales d l'Institut Henri Poincaré 7, reprinted in Studies in Subjective Probability, pp. 950157, Kyburg, Smokler eds., Wiley (1937)

[10] FISCHER, R. W., “Theory Selection in Modal Epistemology”, American Philosophical Quarterly (forthcoming)

[11] VAn FraAssen, B., Laws and Symmetry, Oxford: Clarendon Press (1989)

[12] Goldman, A., "Discrimination and Perceptual Knowledge", Journal of Philosophy 73, pp. 771-791 (1976)

[13] Goodman, N., Fact, Fiction and Forecast, fourth edition, Harvard University Press (1983)

[14] Hanrahan, R. R., “Consciousness and Modal Empiricism”, Philosophia 37, pp. 281-306 (2009)

[15] Harman, G. H., "Inference to the Best Explanation", The Philosophical Review 74 (1), pp. $88-95(1965)$

[16] Hartl, P., "Modal Scepticism, Yablo-style Conceivability and Analogical Reasoning", Philosophical Studies 193 (1), pp. 269-291 (2016)

[17] Hawke, P., "Van Inwagen's Modal Skepticism”, Philosophical Studies, 153 (3):351-364 (2011)

[18] Kelly, K., The Logic of Reliable Inquiry, Oxford University Press (1996).

[19] Kratzer, A., "What Must and Can Must and Can Mean", in Modals and Conditionals, Oxford University Press (2012) 
[20] KRIPKE, S., Naming and Necessity, Blackwell Publishing (1980)

[21] Kung, P., "On the Possibility of Skeptical Scenarios”, European Journal of Philosophy 19 (3), pp. 387-407 (2011)

[22] VAn Inwagen, P., “Modal Epistemology”, Philosophical Studies 92, pp. 67-84 (1998)

[23] LEWIS, D., Counterfactuals, Blackwell (1973)

[24] LEWIS, D., On the Plurality of Worlds, Blackwell (1986)

[25] Norton, J. D., “A Little Survey of Induction”, in Scientific Evidence: Philosophical Theories and Applications, pp. 10-34, Achinstein ed., Johns Hopkins University Press (2005)

[26] Putnam, H., “Meaning and Reference”, Journal of Philosophy 70, pp. 699-711 (1973)

[27] Roca-Royes, S., "Conceivability and De Re Modal Knowledge", Noûs 45 (1), pp.22-49 (2011)

[28] Sonmes, S., Reference and Description. The Case Against Two-Dimensionalism, Princeton University Press, (2005)

[29] Sturgeon, S., “Apriorism About Modality”, in Modality: Metaphysics, Logic and Epistemology, Hale B. and Hoffmann A. eds., Oxford University Press (2010)

[30] Vickers, J., "The Problem of Induction", on-line at the Stanford Encyclopedia of Philosophy, URL: http://plato.stanford.edu/entries/induction-problem/, first published Wed Nov 15, 2006; substantive revision Fri Mar 14, 2014 (2006)

[31] Yablo, S., "Is Conceivability a Guide to Possibility?", Philosophy and Phenomenological Research, 53 (1), pp. 1-43 (1993) 


\section{A Appendix: Hume's problem is live}

I briefly address the concern that the project of replying to Hume's problem has run its course, drawing out, in particular, some considerations that point in the direction of a solution along the lines of that in the current paper. This concern arises by accepting one of the following: (i) there is little reason to think that we actually use enumerative induction in ordinary or scientific contexts; (ii) enumerative induction is a narrow and potentially misleading special case of a broader inference method such as statistical induction or abduction; or (iii) the problem of accounting for enumerative induction has effectively already been solved.

In reply to (i): I believe that enumerative induction plays such a basic role in everyday and scientific reasoning that it is easily overlooked. I reasonably expect that when I place my coffee mug on this table that the mug will not pass through the surface of the table and smash to the floor. This belief, it seems, is not the product of, say, acceptance of a certain theory of the physics of tables and mugs. My grounds are simpler: I have had ample experience in interacting with like objects and project these lessons to future interactions. Further, we can borrow from Norton a prototypical scientific belief that is naturally ascribed to enumerative induction: "we believe all electrons have a charge of $-1.6 \times 10^{-19}$ Coulombs, simply because all electrons measured so far carry this charge" [25, pg.12].

In reply to (ii): I delay discussion of the possibility that enumerative induction can be folded into abduction and concentrate on the exceedingly plausible claim that enumerative induction is a special case of statistical induction (or perhaps just superseded by it). Statistical reasoning has reached a level of sophistication that suggests that undue attention to universal inference might be misleading or limiting. What justifies a focus on simple enumeration?

In response, I suggest the simpler setting of universal inference brings into focus an aspect of the problem of induction that is commonly overlooked - not least in technically sophisticated discussions focused on statistics. 
To illustrate: suppose we embark on a study of the color features of the actual population of emeralds. Before gathering data, we agree (for whatever reason) to take seriously three possible candidate colors: green, yellow and mauve. As it happens, we then observe a random sample of emeralds with these color-types distributed in a 70-30-0 ratio. Statistical induction directs us to project this make-up onto the entire population of emeralds, and we draw our conclusions accordingly. Altogether, our investigative methods raise two obvious questions, though the first receives less attention: what, if anything, justifies our treating green, yellow and mauve as comprising the complete set of possible candidate colors? (In particular, in retrospect, was it sensible to include mauve as a possibility to be ruled out? If so, what justified limiting ourselves to only these three colors?) And, secondly, given a set of possible candidates, what justifies projection from a sample to the whole population? (In particular, did we select enough emeralds for our sample, and use a suitable selection technique? How confident should we be in our conclusion, given the size of our sample? Should we reserve some credence for the hypothesis that some mauve emeralds are lurking out there?)

A focus on enumerative induction simplifies the statistical issues and allows us to focus better on the first, neglected question. Indeed, as should become apparent, the response to Humean skepticism I propose in this paper depends on a view as to how best to answer the first question, not the second.

In reply to (iii): ingenuity aside, there is room to feel dissatisfied with the existing major proposals for dealing with Humean skepticism. I cannot back this claim comprehensively here, so offer only cursory worries for a few promising and influential recent proposals 28

Some propose that enumerative induction is a special case of abductive reasoning. According to the abductivist: the reason to believe that all emeralds are green - given an uninterrupted parade of observed green emeralds - is that this fact, if true, would best explain the observational data [15].

\footnotetext{
${ }^{28}$ Not only cursory, but patently inadequate without a fuller dialectic: the views under consideration have been developed in rich and sophisticated ways by the cited authors, and are not to be dismissed off-hand.
} 
One reason to hesitate to embrace this proposal is skepticism that a happy outcome is likely for the debate concerning the nature of explanation and good explanation [11, 142-148]. However, even for those optimistic about securing the foundations of abduction (such as myself) there is room to doubt that abduction is the antidote to Humean skepticism. A good explanation, it is natural to presume, is one that, in some sense, generates understanding. But it is hard to see how the truth of "all emeralds are green" can by itself generate understanding as to why the emeralds I have observed are green. "All emeralds are green" entails that every emerald I observe is green, but fails to locate this fact in an "illuminating story" involving diverse (and possibly more primitive) facts, laws or entities. Or perhaps the abductivist's claim is that we first use abduction to infer that the underlying causal-structural constitution of an emerald necessitates a green hue, then from this intermediate conclusion infer that all emeralds are green. But if an explanation citing necessitating constitutive facts is fleshed out as a detailed proposal, then it is no longer plausible that the proposed abduction captures the simplicity of enumerative induction; and if the details are left unspecified then we are again left with the question as to whether this "explanation" has genuine explanatory power. Further, the abductivist approach to enumerative induction highlights peculiar mysteries that might seem no better than (or are perhaps equivalent to) the problem of induction. For instance, if simplicity and explantory scope are offered as reasons to accept the hypothesis that all emeralds are green, then this merely provokes the question as to why these factors ought to be taken as a mark of truth.

Next consider the proposal that enumerative induction is simply a matter of subjective Bayesian inference. By subjective Bayesian lights, a rational agent is one whose degrees of belief (credences) take the form of a probability function, and who updates her beliefs by way of Bayesian conditionalization. The Bayesian may then cite a famous result due to De Finetti [9]: in the long run, so long as the agent's prior credence function satisfies certain symmetry properties (namely, a property that goes by the name of exchangeability), a Bayesian agent's credence function will eventually come to 
reflect the frequency information captured in the stream of her observational data. That is: if green emeralds occur with a 100 percent frequency in the observations she collects, she will converge to a credence of 1 with respect to the hypothesis that all emeralds are green - essentially capturing the mechanism of enumerative induction. But this proposal again leaves room for reasonable doubt [8]. The subjective Bayesian does not typically offer reasons to think that a rational agent must have an exchangeable credence function. Nor does the typical subjective Bayesian offer much to guarantee an efficient rate of convergence to inductive conclusions. Yet, intuitively, enumerative induction is a highly efficient form of reasoning. To reasonably conclude that all electrons have a charge of $-1.6 \times 10^{-19}$ Coulombs does not, it seems, require excessive individual measurements.

Finally, consider the proposal of the formal learning theorist [18]: there is no guarantee that the method of enumerative induction arrives at a true conclusion in the short term, but in a certain sense it is guaranteed to hit upon the truth in the long term, thereby rendering it a rational strategy for belief acceptance. For either there are only green emeralds, and so the conclusion "all emeralds are green" is true; or, if indeed there is a yellow emerald out there - and one is guaranteed to eventually observe this fact - then eventually one will come to modify one's incorrect belief. The difficulty with this proposal is, however, two-fold. First, for ordinary agents, it is unlikely that their investigation will be so thorough as to guarantee that, if there is an exception to a universal claim, it will be eventually found. Second, that it is in the long-run strategic to accept a certain claim does not by itself make it likely that that claim is true (as the formal learning theorist admits). So it is left somewhat mysterious for the formal learning theorist why an agent should believe the conclusion of a universal inference, as opposed to merely accept it for strategic purposes. 